\title{
Electromagnetic Wave and Gaseous Communication between Individuals
}

\author{
Kong Derick Njikeh \\ Faculty of Medicine and Biomedical Sciences, University of Yaounde I, Yaounde, Cameroon \\ Email: kongderick@yahoo.com
}

Received 11 December 2013; revised 10 January 2014; accepted 8 February 2014

Copyright (C) 2014 by author and Scientific Research Publishing Inc.

This work is licensed under the Creative Commons Attribution International License (CC BY). http://creativecommons.org/licenses/by/4.0/

(c) (i) Open Access

\begin{abstract}
The brain produces an electromagnetic wave throughout the day and the strength of this wave depends on the activity of the brain. It is said that this wave is a weak wave which does not cover a greater distance. But I do believe that it is like the radio wave in the distance it covers and frequency it possesses. I do believe that there is a communication between individuals through electromagnetic wave (brain wave) and a gas (Nitric Oxide) produced by the brain. This can be observed in our daily experiences. This can also help us understand that as a computer can receive and record the brain waves, so an individual can also receive and integrate these waves and it is being decoded to release the message it contains. In this respect, it can be said that the state of consciousness in an individual can be understood by another through concentration.
\end{abstract}

\section{Keywords}

Telepathy; Electromagnetic Wave; Encoded; Brain Wave; Consciousness; Decoded

\section{Introduction}

I do believe that there is a system of communication between individuals through an electromagnetic wave (brain wave) and a gas (Nitric Oxide) produced by the brain. Demonstrations by theoretical arguments show that this electromagnetic wave is the origin of consciousness and that information has been encoded in this wave [1]. This ability of communication can also help us understand that, as a computer can receive and record the brain wave, so an individual can also receive and integrate this wave and it is being decoded to release the message encoded in it. In this respect, it can be said that the state of consciousness in an individual can be understood by another through concentration.

Some theorists claimed that this means of communication through electromagnetic wave is not possible because the brain wave produced is very weak and cannot travel greater distances. They put up the augments that, 
brain-waves are too weak to explain Telepathy [2], and also the electromagnetic field out of the head is far too weak and highly unlikely that any other brain could detect it, and still more unlikely that the other brain could decode the EM field information that was encoded by your brain.

It was proposed that extremely low-frequency (ELF) electromagnetic waves may be able to carry telepathic and clairvoyant information. I also do think that this electromagnetic wave has a very low frequency similar to/or lower than that of a radio wave, capable of transmitting information from one individual' brain to another regardless of the distance.

\section{Production of Brain Wave and the Process of Communication}

When an individual is in thoughts, action potentials are being generated simultaneously in thousands of neurons due to the passage of ions $\left(\mathrm{Na}^{+}\right)$across the neuronal membrane [3]. These action potentials are being propagated in the form of impulses (electric current) through the neurons. Due to the propagation of these impulses, there is the induction of a magnetic field which is perpendicular to the direction of the electric field created by these impulses [4]. The present of both fields creates an electromagnetic wave that contains the coded information of the thoughts (state of consciousness) called the brain wave. This wave is then transmitted out of the brain into space where it is being received by the neurons of another individual [5]. It is then decoded to produce the original impulse containing the same information. This can act as an explanation to the phenomenon of telepathy (thought-transference). The strength of this wave depends on the degree of consciousness and the transmission from one individual to another is time dependent, called the lag time.

\section{Observations}

This means of communication was observed between two students in a classroom who were bench mates. An unfamiliar question was asked, one of the students thought of the respond and a few seconds later, the mate also had a respond which was exact to his. This is associated to latent telepathy which is described as the transfer of information, through psi, with an observable time-lag between transmission and reception. It was realized that, this happened in many occasions and with different set of students who were together.

Some individuals have a specific conscious mind capable of receiving information from far away distances from those who are dear to them. This can be observed in some cases where; certain individuals of a family, who are far away from the family house, can feel the presence of, a mention of their names or the dead of a family member. This is associated to intuitive telepathy which is described as the transfer of information, through psi, about the present state of an individual's mind to another individual.

It is observed that a greater percentage of a steady population in a vehicle in motion always get asleep. This sleeping state is always induced by a few individuals and the message is being transferred to others.

A mixture of intelligent students and less intelligent students in a group reading which is being characterized by no physical communication, results in the improvement of intelligence in the less intelligent students.

\section{Gaseous Communication}

During impulse propagation in the neurons of the brain, at the level of the synaptic knob of specific neurons, there is the release of Nitric Oxide which is a neurotransmitter. This Nitric Oxide then diffuses into the blood capillaries surrounding the neurons and it is transported to the lungs where it diffuses into the Alveoli and it is expired. The presence of this gas in the outer space influences the thought or state of consciousness of the individual around the area covered by the gas. This is done through inspiration of the gas which is transported to the brain by the circulatory system where it acts as a neurotransmitter to the neurons specific to it and produces the same thoughts. Much is not to be said about this means of communication because the quantity of gas produce and the area covered are mild.

\section{Production of Click Sounds in the Brain and Flashes of Lights in the Eyes}

The neurons in the brain are connected to each other through specific junctions called synapses. Each synapse contains a potential space called the synaptic cleft that separates the postsynaptic and presynaptic membranes. During hyperactivity of the brain, there is rapid and intense generation of impulses. The propagation of these 
impulses leads to intense vibration of the molecular components of the neuronal membrane which leads to the sliding of weakly attached neurons from their junctions on to other neurons. This contact then leads to the production of sparks. These sparks causes direct stimulation of afferent neurons that leads to the visual field of the occipital lobe. Man is aware of the sparks through impulses transmitted to the retina and being represented as star-like or sharp light.

At the instance of light production, there is also sound production due to the vibration of the molecular components of the neurons and air in the synaptic space. These sounds directly stimulate specific afferent neurons and impulses are being transmitted to the audible field in the temporal lobe. It was also explained that brain noise is been generated by conducting neurons [6]. These explanations can be observed in many situations such as:

* An impact of the head on a hard surface causes star-like light sensation and sharp sound production.

* High concentration on a particular situation for a while is accompanied by flash light sensation with a sharp sound in the brain.

* Epileptic patients having flash light sensation before going into seizure.

\section{Concentration and Epilepsies}

A highly concentrated individual in thoughts produces a strong wave and a gaseous neurotransmitter. This is then received by neurons of another individual and that same process is induced in him. If the receiver of this wave have low neuronal activity threshold compared to the producer, the impulses generated will lead to hyperactivity of his neuronal system resulting to the manifestation of epileptic signs. This can explain one aspect of the metaphysical etiology of epilepsy.

I do believe that individuals with low brain activity such as the layman, have a very high probability of developing epilepsy than those with high brain activity such as scientists. This can suggest that the management of epilepsies can be improved by increasing the individuals' brain activity by subjecting him to concentrated needed activities in which his neuronal system will adapt with time.

\section{Experimental Evidence}

There are some evidences that neurons do communicate between individuals through electromagnetic wave. An experiment was conducted in the 1930s to explain this mechanism [7]. In this experiment, the "sender" looks at a series of cards while the "receiver" guesses the symbols. Also another experiment was conducted which consisted of two individuals at the end of each day to relax and visualize a mental image or "thought impression" of the events or thoughts they had experienced in the day and then to record those images and thoughts on paper in a diary. The results at the end when comparing one individual' diary to another's, were that "seventy-five percent were found to be correct". From these, the floor was opened for further experiments to be developed in this field.

Man can only use $100 \%$ of his brain when he knows everything in the world that he lives in. This will never happen because the more he searches to know, the more problems he creates and the more answers he needs. Therefore only our creator will forever have a complete knowledge of his creation.

\section{References}

[1] McFadden, J. (2002) Evidence for an Electromagnetic Field Theory of Consciousness. Journal of Consciousness Studies, 9, 23-50.

[2] Myers, F.W.H. (1882) Telepathy.

[3] Guyton and Hall (2006) Motor and Integrative Neurophysiology. 11th Edition, Saunders/Elsevier, Philadelphia.

[4] Serway, R.A. and Jewett, J.W. (2013) Electromagnetic Waves. In: Physics for Scientists and Engineers 6E, Cengage Learning, Stamford.

[5] Lee, F. (2012) The Mechanism of Wireless Communication in the Brain. http://www.web-books.com/eLibrary/ON/B0/B98/06M98.html

[6] Saliu, I. (2003) Brain Waves, Communication, Body Language, Behavior. http://saliu.com/brain.html

[7] Rhine, J.B. and Pratt, J.G. (1940) Extra-Sensory Perception after Sixty Years. Henry Holt, New York. 